\title{
Utilization of a hollow copper cathode laser in producing polymers
}

\author{
N. A. Al Muslet ', W.S.A. Rahman ${ }^{1}$, M. Alkareem ${ }^{2}$ \\ ${ }^{1}$ Institute of Laser; ${ }^{2}$ Dept. of Chemistry, College of Science, Sudan University of Science \& Technology, Sudan. \\ mnmfa2008@yahoo.com
}

Abstract: A Pulsed, home- built, hollow copper cathode discharge laser (HCCL) in the visible region and $1.76 \mathrm{mj}$ energy per pulse, was used to irradiate a monomer so as to produce polymer. Three different categories of samples were irradiated for different intervals of time: 30 , 60 and 90 minutes. Infrared Spectrometry was carried for the samples before and after irradiation and the collected spectra were analyzed and discussed. The results showed that the polymerization process starts in a very short time $(30 \mathrm{~min})$, which indicates the efficiency of this type of laser in such application.

Keywords: Laser, hollow copper cathode, polymerization.

Theory

Lasers are considered being as one of the most significant tools in chemistry for its remarkable characteristics, which are: narrow spectral range, high intensity and the possibility of producing pulses of very short duration. Lasers can be used to drive chemical reaction in a chosen direction or to initiate reactions instead of the traditional method of adding thermal energy. Also, another area of laser - enhanced chemistry is the systemization of reactions by the excitation of species that it dose not change. In addition to that, they are capable of monitoring the dynamics of the chemical reactions and polymers production (John F. Ready, 1997).

A Polymer is a large molecule comprised of repeated structural units. These structural units are much related to the starting material from which the polymer is originated (Monomer).

The Degree of Polymerization ( $n$ ), is the average number of monomer units that have polymerized together. It depends on the nature of the monomer and the conditions under which they formed (Fred \& Bill Meyer, 1971). Some examples of the structures of polymers and monomers are shown in Table 1, (Malcolm P. Stevens, 1984).

The physical properties of a polymer material are largely determined by: molecular weight, strength of intermolecular forces, regularity of the polymer structure, flexibility of the polymer molecule.

Polymers are classified according to many parameters such as the origin, the physical properties, and how the structural groups are joined. According to the origin, polymers are classified into natural and synthetic

Table. 1. Structures of some monomers and Polymers

\begin{tabular}{|l|l|l|l|}
\hline \multicolumn{1}{|c|}{$\begin{array}{c}\text { Monomer } \\
\text { Structure }\end{array}$} & \multicolumn{1}{|c|}{$\begin{array}{c}\text { Monomer } \\
\text { Name }\end{array}$} & \multicolumn{1}{c|}{$\begin{array}{c}\text { Polymer } \\
\text { Repeating unit }\end{array}$} & \multicolumn{1}{c|}{$\begin{array}{c}\text { Polymer } \\
\text { Name }\end{array}$} \\
\hline $\mathrm{CH}_{2}=\mathrm{CH}_{2}$ & Ethylene & {$\left[\mathrm{CH}_{2} \mathrm{CH}_{2}\right]$} & Polyethylene \\
\hline $\mathrm{CH}_{3} \mathrm{CH}=\mathrm{CH}_{2}$ & Propylene & {$\left[\mathrm{CH}_{2}-\mathrm{CH}-\mathrm{CH}_{3}\right]$} & Polyproplyene \\
\hline $\mathrm{F}_{2} \mathrm{C}=\mathrm{CF}_{2}$ & Tetrafluoroethylene & {$\left[\mathrm{CF}_{2} \mathrm{CF}_{2}\right]$} & Polytetrafluoroethylene \\
\hline
\end{tabular}

Research article

Clndian Society for Education and Environment (iSee)

"Laser"

http://www.indjst.org polymers. According to the physical properties, are classified into: elastomers, fibers and plastics: when considering the functional group, polymers are classified into (a)linear, (b)branched and (c)net- work or crosslinked polymers as shown in Fig.1 (Malcolm P. Stevens, 1984).

Polymerization process can be preceded by many chain reactions. It may be subdivided into many types, such as free radical addition polymerization, an ionic addition polymerization, and cationic polymerization. A simple picture for the dynamic of polymerization by free radical addition is on the following steps:

Initiation

Free radical activates the monomer by opening the double bond.

Initiation steps are as follows:

- The initiator (I) decomposed into free radical $\left(R^{*}\right)$ : Initiator (I) $\rightarrow \mathrm{R}^{*}$

- The second step; the free radicals are added to the monomer unit $\mathrm{M}$ to form a chain radical $\mathrm{M}_{1}$ *

$\mathrm{R}^{*}+\mathrm{M} \rightarrow \mathrm{Mi}^{*}$

where $k_{d}, k_{i}$ are the speed constants for decomposition and initiation of the previous reactions.

Propagation

The chain radical formed in the previous step is capable of adding successive monomers to propagate the chain as

follows:

$$
\begin{aligned}
& M_{1}{ }^{*}+M \rightarrow M_{2}{ }^{*} \\
& M_{2}{ }^{*}+M \rightarrow M_{3}{ }^{*} \\
& \left.M_{x}{ }^{*}+M \rightarrow M^{*}{ }^{*}+1\right)
\end{aligned}
$$

where $k_{p}$ is the speed constant for the propagation.

Termination

The process of propagation can keep in continuing until the supply of monomers is finished. Termination process can take place in two ways:

- The coupling of two radicals:

$$
\mathrm{M}_{\mathrm{x}}{ }^{*}+\mathrm{M}_{\mathrm{y}}{ }^{*} \rightarrow \mathrm{M}_{(\mathrm{x}+\mathrm{y})}
$$

- Two radical undergo disportionation:

$M_{x}^{*}+M_{y} * \underset{k_{t c}}{\stackrel{k_{\text {td }}}{\rightarrow}} M_{x}+M_{y}$

where $k_{t c}, k_{t d}$ are the speed constants for the termination by coupling and disportionation reactions, (Burnett, 1954).

respectively

Free radical polymerization, for some monomers, can be obtained by a photochemical system (Malcolm P. Stevens, 
1984). Some photochemical systems are capable of producing free radical polymerization, for some monomers.

Lasers have been utilized to activate some organic dyes that have selective absorption for the laser light at a certain range of wavelengths, and then it will be excited to a higher state. At this excited state it becomes strong oxidizer, capable of participating in a redox reaction with mild reducing agent (Zare et al., 1995). Then this reaction results in generation of free radicals that ready to attack the monomer which leads to homoletic opening of the double bond.

That mean formation of radicals chain reaction and the reaction propagates, and hence the polymer is produced. An example will be given below:

$\begin{array}{cc}{ }^{1} \mathrm{MB}_{0} & \text { hv } \\ \text { Excited single } \\ \text { state } & { }^{1} \mathrm{MB}^{+} \\ \text {Excited single } \\ \text { state }\end{array}$

In forming the Poly Acrylamide polymer, a Methylene Blue dye is used as strong oxidizer. The mechanism of the reaction is as follows:

A mild reducing agent (triethanolamine $\mathrm{NR}_{3}$ ) is added,

${ }^{3} \mathrm{MB}^{+}+\mathrm{NR}_{3} \rightarrow \mathrm{MB}^{-}+\mathrm{NR}_{3}{ }^{+}$

This photochemical redox reaction results in generation of two radicals. Now the radicals are ready to attack the Acrylamide molecules and the polymerization reaction propagates to produce the monomer.

Experimental work

A home built hollow cupper cathode discharge laser was used to irradiate samples prepared of mixture of monomers and other reactants. Analytical grade of reagents was used to prepare the reactants. The IR spectra of the irradiated samples, with different intervals of time, were collected using infrared spectrometer. The specifications of the laser which was used as a source of irradiation are listed in Table 2.

For the sample preparation, $15 \%$ gelatin solution was prepared as follows:

$15 \mathrm{~g}$ of gelatin was weighted and added to $10 \mathrm{ml}$ of hot $\left(50-60{ }^{\circ} \mathrm{C}\right)$ distilled water in a conical flask $(100 \mathrm{ml})$. The gelatin was dissolved and the volume was adjusted by addition of the hot water to $100 \mathrm{ml}$. The solution was kept warm using a magnetic stirrer ready for use.

Second, the mixture of the reactant was prepared as: $15.57 \mathrm{~g}$ of the monomer (acrylamide) was weighted and added to $0.025 \mathrm{~g}$ of methylene blue and $3.3 \mathrm{ml}$ of
Table 2. The hollow copper cathode laser parameters

\begin{tabular}{|l|l|}
\hline Active medium & Cupper ions \\
\hline Wavelength & $513.5 \mathrm{~nm}$ \\
\hline Gas carrier & Helium \\
\hline Helium pressure & $28 \mathrm{mbar}$ \\
\hline Discharge current & $1.96 \mathrm{Amper}$ \\
\hline Temporal mode & Pulsed \\
\hline Output energy & $1.76 \mathrm{~mJ}$ \\
\hline Pulse repetition rate & $0.675 \mathrm{kHz}$ \\
\hline Pulse duration & $1.4 \mathrm{msec}$ \\
\hline
\end{tabular}

tryethanolamine in $100 \mathrm{ml}$ volumetric flask. The volume was adjusted to 100 $\mathrm{ml}$ using the $15 \%$ gelatin solution, and then well mixed.

One drop from the prepared reactants mixture was applied on clean microscopic slide. The diameter of each sample was $5 \mathrm{~mm}$ approximately. These samples were exposed to the laser radiation for different intervals of time $(1 / 2,1$, and $1 \frac{1}{2}$ ) hours. Some samples were kept as a control, i.e. not exposed to the radiation. The beam was focused so that the entire volume of the sample was uniformly irradiated as shown in Fig.2. The beam was reflected by a plane mirror downwards perpendicularly on the sample.

Note that the process of irradiation was completed in a dark room to avoid any source of illumination except the laser beam that may affect the polymerization process.

The irradiated samples were subjected to spectroscopic measurements by an infrared spectrometer. The spectra of the samples indicate whether the polymer is produced or not.

The sample was scratched from the slide and grounded finely in a mortar. Then a $0.5 \mathrm{mg}$ of a $\mathrm{KBr}$ powder (analar) was added to the sample in the mortar. The mixture was transferred to the compressing machine and pressed (8 Tons) to form a transparent disk. The disk was mounted in a suitable holder and kept in a desiccator to avoid moisture.

The spectra of the samples were collected using IR Spectrometer (Thermo- Nicot) in the wavenumber range (500-4000) $\mathrm{cm}^{-1}$ with resolution of $4 \mathrm{~cm}^{-1}$.

\section{Results and discussion}

The action of the high focused energy of the laser beam is to breakdown the strong covalent bonds $\mathrm{C}=\mathrm{C}$, which normally characterize the

monomers, and subsequently allows another molecule to be attached. Furthermore, it speeds up the polymerization reaction rate. The results of the obtained spectra are as follows:

\section{IR Spectrum of the mixed reactants of polymerization} before Irradiation:

The I.R spectrum of the mixture of reactants, comprising the polymerization mixture, is depicted in Fig.3. The mixture reactants include the monomer, the oxidizing agent and the reducing agent in a gelatin 
Table 3. Spectral data of the mixed reactants before irradiation

\begin{tabular}{|c|c|c|c|}
\hline $\begin{array}{c}\text { Present work } \\
\text { band position } \\
\left(\mathrm{Cm}^{-1}\right)\end{array}$ & $\begin{array}{c}\text { Previous work } \\
\text { (Elameer,2005) } \\
\text { band position }\left(\mathrm{Cm}^{-1}\right)\end{array}$ & $\begin{array}{c}\text { Reference data } \\
\text { (James et al., 1988) } \\
\text { band position }\left(\mathrm{Cm}^{-1}\right)\end{array}$ & $\begin{array}{c}\text { Probable } \\
\text { assignment }\end{array}$ \\
\hline 1658.74 & 1675 & $1630-1690$ & $\mathrm{C}=\mathrm{O}$ stretch \\
\hline 3359.84 & 3425 & $3200-3400$ & $\mathrm{~N}-\mathrm{H}$ stretch \\
\hline 1567.77 & 1620 & $1560-1690$ & $\mathrm{C}=\mathrm{C}$ stretch \\
\hline 1454.21 & 1425 & $1450-1575$ & $\mathrm{~N}-\mathrm{H}$ bend \\
\hline 2924.40 & 2950 & $2850-2960$ & $\mathrm{C}-\mathrm{H}$ stretch \\
\hline
\end{tabular}

solution. The experimental data of the band positions are compared with a previous study as illustrated in Table 3 and with referenced data in correspondence of its probable assignment. Only the major vibration frequencies are listed here. The obtained results from a $\mathrm{KBr}$ disc of the mixture spectrum shows an agreement with the referenced data (Table 3).

IR spectrum of mixed reactants after laser irradiation for 30 min (Fig.4):

The degradation of the bond $\mathrm{C}=\mathrm{C}$ which considered as a significant criteria of the monomer, nearly disappeared. It means that the polymerization reaction

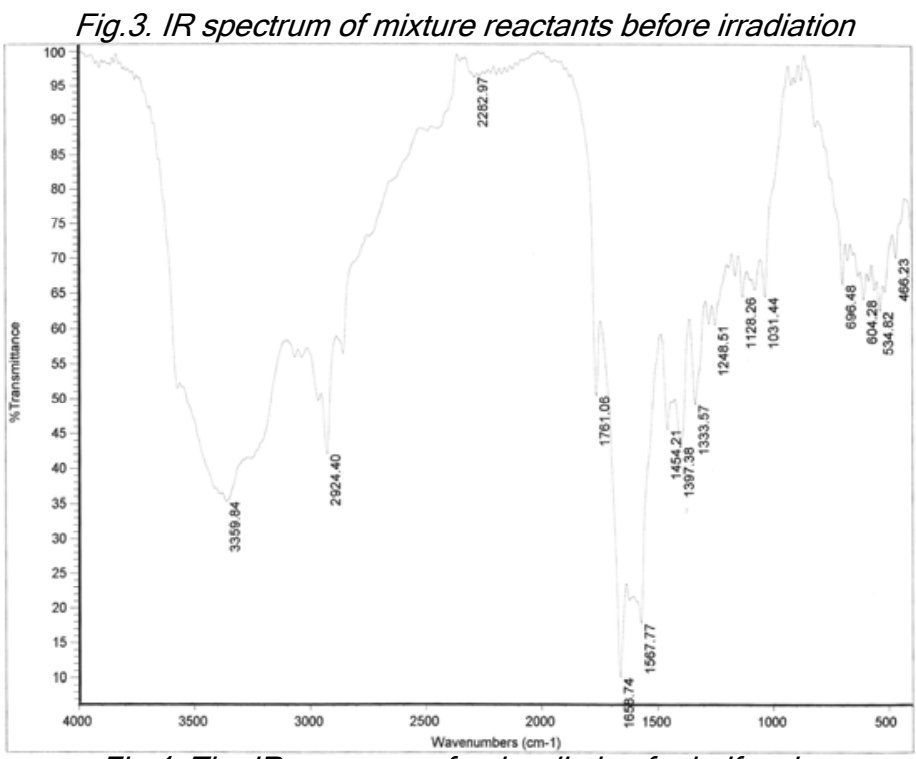

Fig.4. The IR spectrum after irradiation for half an hour

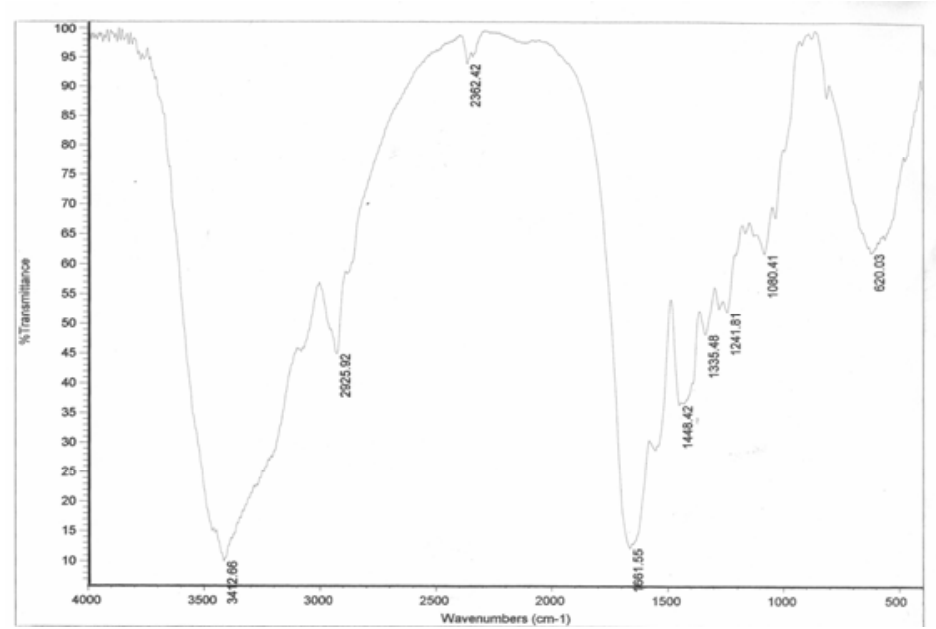

become obvious after $30 \mathrm{~min}$.

The data collected from the spectrum of a $\mathrm{KBr}$ disc of mixture reactant irradiated for half an hour using the hollow cathode discharge laser are listed in Table 4. The results obtained in the present work are compared with a previous data where the irradiation was done with the helium- neon laser for the same mixed reactant and irradiation time of 36 hours. The data shows a good agreement with both previous work (Elameer, 2005) and that data obtained from reference (James D. Ingle \& Crouch, 1988) although the irradiation time of the present

Fig.5. a. The IR spectrum after irradiation for 1 hour.

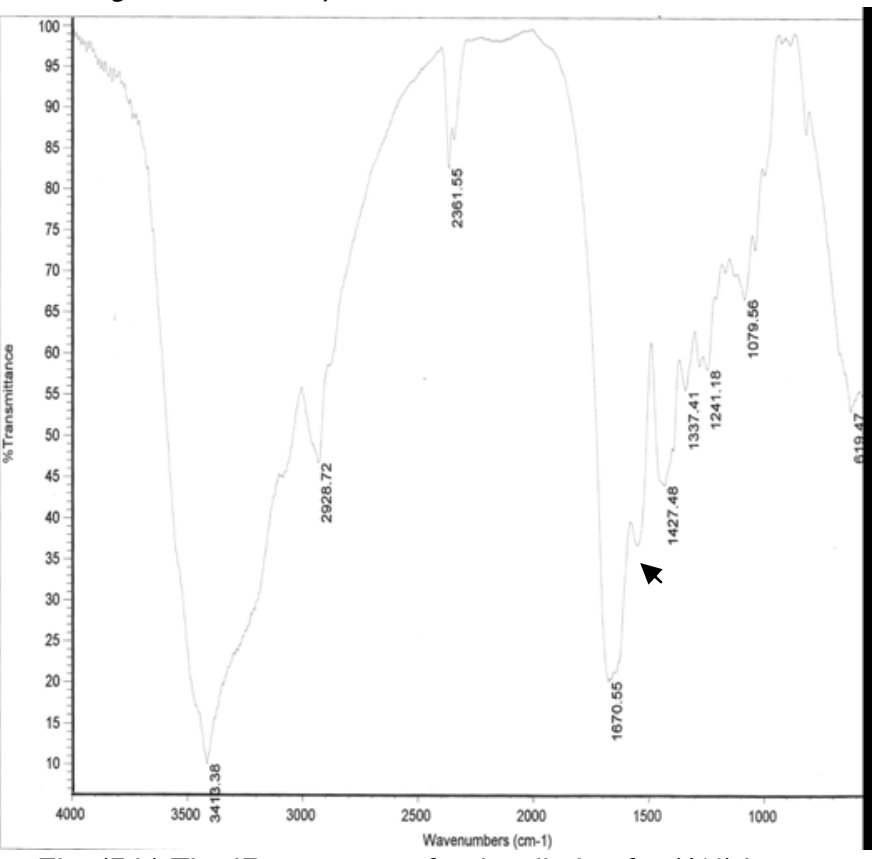

Fig. (5.b) The IR spectrum after irradiation for (11/2) hour

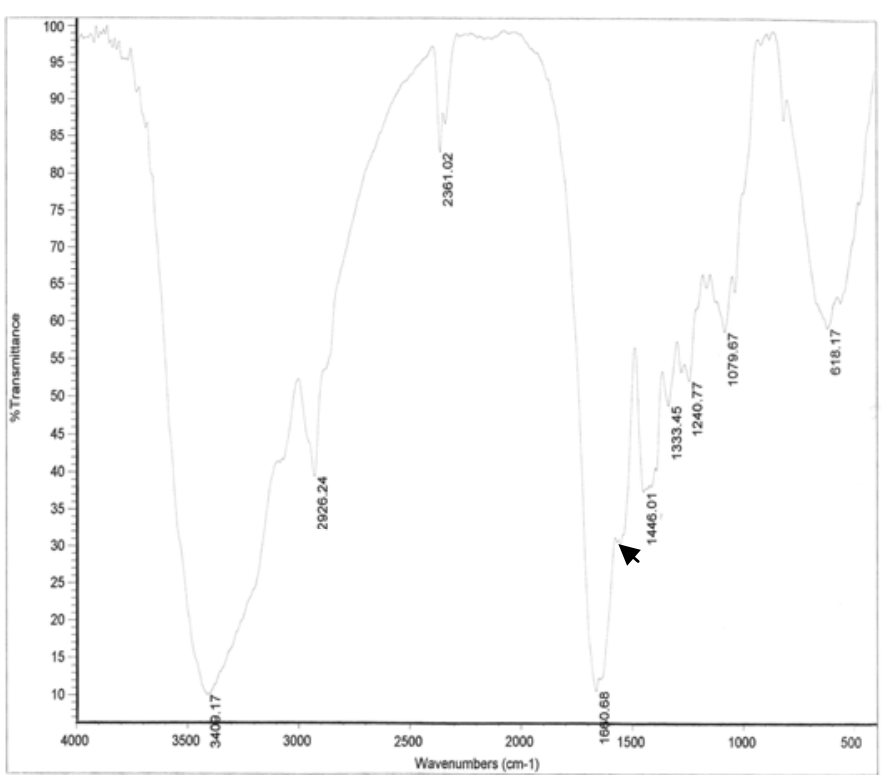

Laser" http://www.indjst.org
Muslet et al. Indian J.Sci.Technol. 
work is just $30 \mathrm{~min}$. The band position $3413.38 \mathrm{~cm}^{-1}$ is slightly out of the range and that may be due to the laser beam direction which makes a new orientation as a result of polarization.
2. Elameer $A E$ (2005) Production of polyacrylamide from monomer using $\mathrm{He}-\mathrm{Ne}$ laser. MSc. Thesis, Laser Institute, Sudan University of Science and Technology.

Table. 4. Comparison of obtained data with that of previous work \& the referenced

\begin{tabular}{|c|c|c|l|}
\hline $\begin{array}{c}\text { Present work } \\
\text { band position } \\
\left(\mathrm{Cm}^{-1}\right)\end{array}$ & $\begin{array}{c}\text { Previous work } \\
\text { (Elameer,2005) } \\
\text { band position }\left(\mathrm{Cm}^{-1}\right)\end{array}$ & $\begin{array}{c}\text { Reference data } \\
\text { (James } \text { et al., 1988) } \\
\text { band position }\left(\mathrm{Cm}^{-1}\right)\end{array}$ & $\begin{array}{c}\text { Probable } \\
\text { assignment }\end{array}$ \\
\hline 1658.74 & 1673 & $1630-1690$ & $\mathrm{C}=\mathrm{O}$ stretch \\
\hline 3393.38 & 3384 & $3200-3400$ & $\mathrm{~N}-\mathrm{H}$ stretch \\
\hline 1427.84 & 1423 & $1450-1575$ & $\mathrm{~N}-\mathrm{H}$ bend \\
\hline 2928.40 & 2925 & $2850-2960$ & $\mathrm{C}-\mathrm{H}$ stretch \\
\hline
\end{tabular}

3. Fred W. and Bill Meyer (1971) Polymer science, Wiley interSciences, John Wiley \& Sons Inc., Second Edition, New York.

4. James D. Ingle and J.S Crouch (1988) Spectrochemical Analysis, Prentice -Hall, Inc. First edition.

5. John F. Ready (1997) Industrial application of lasers, Academic

Fig.6. The effect of the exposure time on the polymerization rate

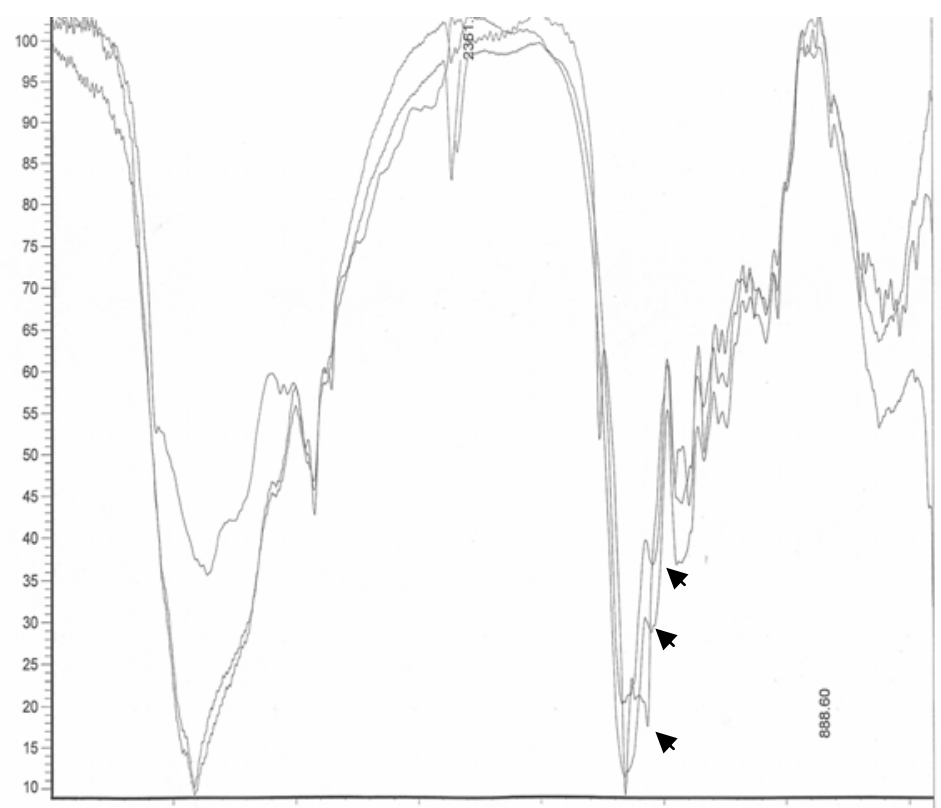

Press, Second edition.

6. Malcolm P. Stevens (1984) Polymer chemistry. Basra University Press, Iraq.

7. Zare RN et al. (1995) Laser experiments for beginners, University Science Books.

The effect of exposure time on rate of polymerization:

After the successful experiment of producing polymer from monomer using the technique of $\mathrm{HCCL}$ irradiation, other samples of the mixture reactants were exposed to different irradiation time: 1 and $1 \frac{1}{2}$ hour. The IR spectra that show the transmittance as a function of the wave number are shown in Fig. 5 a,b for 1 hour and $1 \frac{1}{2}$ hour, respectively. The gradual declination of the $\mathrm{C}=\mathrm{C}$ bond can be observed. In other words, increasing the irradiation time increase the polymerization rate. This can be explained easily since the laser energy deposited in mixed reactant is time dependent. Fig. 6 shows the effect of exposure time on the polymerization rate. In our view, this rapid change may be due to increase of photon energy since the wavelength of the produced laser is $513.8 \mathrm{~nm}$ which is greater than the $\mathrm{He}-\mathrm{Ne}$ laser $(632.8 \mathrm{~nm})$. The other factor is the energy per pulse as a result of the pulsed mode of the laser $(1.76 \mathrm{~mJ})$.

\section{References}

1. Burnett CM (1954) Mechanism of polymer reactions.

Wiley - Inter Science, New York. 